\title{
Novel archaeal metagenome assembled genomes from acidophilic microbial community of Parys Mountain copper mine (UK)
}

\author{
Aleksei Korzhenkov \\ Kurchatov genome center, \\ NRC Kurchatov Institute, \\ Moscow, Russia \\ oscypek@ya.ru
}

Peter N. Golyshin

School of Natural Sciences

Centre for Environmental Biotechnology

Bangor University, Bangor, UK

p.golyshin@bangor.ac.uk

\author{
Stepan V. Toshchakov \\ Winogradsky Institute of \\ Microbiology \\ FRC "Biotechnology" RAS, \\ Moscow, Russia \\ stepan.toshchakov@gmail.com \\ Olga V. Golyshina \\ School of Natural Sciences \\ Centre for Environmental \\ Biotechnology \\ Bangor University, Bangor, UK \\ o.golyshina@bangor.ac.uk
}

\author{
Ilya V. Kublanov \\ Winogradsky Institute of Microbiology \\ FRC "Biotechnology" RAS, Moscow, \\ Russia \\ kublanov.ilya@gmail.com
}

\begin{abstract}
Metagenomic analysis is a powerful tool for discovery of novel microbial taxa. Here we report reconstruction of several archaeal metagenome assembled genomes (arMAGs) belonging to uncultivated lineages of Euryarchaeota and DPANN superphylum.
\end{abstract}

$\underset{\text { metagenomics. }}{\text { Keywords }}-$ extremophiles, acidophiles, archaea,

\section{MOTIVATION AND AIM}

\section{Motivation}

Extremophilic microbial communities represent an inexhaustible source of novel microbial lineages, enzymes, mechanisms of adaptation and evolution. Recent advances in metagenomic analysis techniques allow us the direct analysis of uncultured extremophiles' genomes and predict their ecological niches and metabolic characteristics.

Aim

Our recent work based on NGS metabarcoding techniques revealed prevalence of Euryarchaeota and presence of DPANN archaea in the acidophilic microbial community of Parys Mountain mine (Anglesey Island, UK) [1]. In this study we aimed to acquire genomic sequences of representatives of earlier detected taxa.

\section{Methods}

Metagenomic analysis of acidic mine drainage (AMD) sediment samples was performed as described previously [2]. Metagenomic bins were formed using YAMB [3]. Completeness and contamination of bins were assessed using CheckM [4]. MAG quality score was calculated as genome completeness - $5 \cdot$ genome contamination [5]. MAGs were annotated using RAST web-server with RASTtk pipeline [6]. Preliminary taxonomic assignment of MAGs was performed by aligning $16 \mathrm{~S}$ rRNA gene sequences against NCBI nr/nt and SILVA reference databases. Multilocus phylogenetic analysis was performed using EPhIMM tool (http://github.com/laxeye/EPhIMM/) with a subset of single copy conserved archaeal genes [4]. Average nucleotide identity (ANI) was calculated using ani.rb script (https://github.com/lmrodriguezr/enveomics/). Functional annotation was made using KAAS server [7], metabolic pathways mapping was made using KEG Mapper [8].

\section{Results}

Of 31 reconstructed metagenomic bins eight bins were assigned to Archaea. Six of reconstructed archaeal bins satisfied the high quality MAG criterion - quality score $>50$ [5]. Four of identified MAGs were closely related to previously reported 'Ca. Parvarchaeum acidophilus' ARMAN-5 [9], Cuniculiplasma divulgatum [10], 'Ca. Mancarchaeum acidiphilum' Mia14 [1], and Thermoplasmatales archaeon E-plasma [11] (bin-24, bin-28, bin-31 and bin-r-1, respectively). The other two MAGs show significant remoteness from known genome and MAG sequences on the basis of phylogenetic analysis we could say about relatedness of bin-5 to 'Terrestrial Miscellaneous Euryarchaeal Group' and place bin-30 into cluster with Cuniculiplasma divulgatum and several uncultured Thermoplasmatales archaea such as UBA582 and E-plasma.

TABLE 1. CHARACTERISTICS OF HIGH QUALITY ARCHAEAL MAGS

\begin{tabular}{|c|c|c|c|c|}
\hline ID & $\begin{array}{l}\text { MAG } \\
\text { size, } \\
\text { Mbp }\end{array}$ & $\begin{array}{l}\text { MAG } \\
\text { quality } \\
\text { score }\end{array}$ & $\begin{array}{l}\text { Nearest cultivable archaea } \\
\quad(16 \mathrm{~S} \text { identity, \%) }\end{array}$ & $\begin{array}{c}\text { Nearest } \\
\text { genome/MAG } \\
(\mathrm{ANI}, \%)\end{array}$ \\
\hline bin-r-1 & 1.7 & 88.75 & $\begin{array}{c}\text { Cuniculiplasma divulgatum } \\
\text { strain S5 (93.06) }\end{array}$ & $\begin{array}{c}\text { Thermoplasmatales } \\
\text { archaeon E-plasma } \\
\text { (98.96) }\end{array}$ \\
\hline bin-5 & 1.8 & 78.4 & $\begin{array}{l}\text { Methanomassiliicoccus } \\
\text { luminyensis B10 (83.15) }\end{array}$ & - \\
\hline bin-24 & 0.8 & 67.33 & $\begin{array}{c}\text { Methanococcus aeolicus str. } \\
\text { Nankai-3 (74.63) }\end{array}$ & $\begin{array}{l}\text { 'Ca. Parvarchaeum } \\
\text { acidophilus' } \\
\text { ARMAN-5 (99.21) }\end{array}$ \\
\hline bin-28 & 1.9 & 92.28 & $\begin{array}{l}\text { Cuniculiplasma divulgatum } \\
\text { strain PM4 (100) }\end{array}$ & $\begin{array}{l}\text { Cuniculiplasma } \\
\text { divulgatum strain } \\
\text { PM4 (99.80) }\end{array}$ \\
\hline bin-30 & 2.3 & 93.08 & $\begin{array}{l}\text { Cuniculiplasma divulgatum } \\
\text { strain PM4 (94.85) }\end{array}$ & - \\
\hline bin-31 & 0.9 & 82.4 & $\begin{array}{l}\text { ‘Ca. Mancarchaeum } \\
\text { acidiphilum' Mia14 (100) }\end{array}$ & $\begin{array}{c}\text { ‘Ca. Mancarchaeum } \\
\text { acidiphilum' Mia14 } \\
(99,99)\end{array}$ \\
\hline
\end{tabular}




\section{ACKNOWLEDGMENT}

Supported by a grant of Ministry of Science and Higher Education of Russian Federation allocated to the Kurchatov Center for Genome Research (075-15-2019-1659).

\section{References}

[1] Golyshina, O. V. et al. (2017). 'ARMAN'archaea depend on association with euryarchaeal host in culture and in situ. Nature communications, 8(1), 1-12.

[2] Korzhenkov, A. A. et al. (2019). Archaea dominate the microbial community in an ecosystem with low-to-moderate temperature and extreme acidity. Microbiome, 7(1), 11.

[3] Korzhenkov, A. (2019). YAMB: metagenome binning using nonlinear dimensionality reduction and density-based clustering. BioRxiv, 521286.

[4] Parks, D. H. et al. (2015). CheckM: assessing the quality of microbial genomes recovered from isolates, single cells, and metagenomes. Genome research, 25(7), 1043-1055.
[5] Parks, D. H. et al. (2017). Recovery of nearly 8,000 metagenomeassembled genomes substantially expands the tree of life. Nature microbiology, 2(11), 1533-1542.

[6] Brettin, T. et al. (2015). RASTtk: a modular and extensible implementation of the RAST algorithm for building custom annotation pipelines and annotating batches of genomes. Scientific reports, 5, 8365.

[7] Moriya, Y. et al. (2007). KAAS: an automatic genome annotation and pathway reconstruction server. Nucleic acids research, 35 (supp1_2), W182-W185.

[8] Kanehisa, M., \& Sato, Y. (2020). KEGG Mapper for inferring cellular functions from protein sequences. Protein Science, 29(1), 28-35.

[9] Baker, B. J. et al. (2010). Enigmatic, ultrasmall, uncultivated Archaea. Proceedings of the National Academy of Sciences, 107(19), 8806-8811.

[10] Golyshina, O. V. et al. (2016). Biology of archaea from a novel family Cuniculiplasmataceae (Thermoplasmata) ubiquitous in hyperacidic environments. Scientific reports, 6, 39034.

[11] Dick, G.J. et al. (2009). Community-wide analysis of microbial genome sequence signatures. Genome biology, 10(8), R85. 